\title{
Discovery of multiple ultra-luminous X-ray sources in the galaxy KUG 0214-057
}

\author{
M. G. Watson ${ }^{1}$, T. P. Roberts ${ }^{1}$, M. Akiyama ${ }^{2}$, and Y. Ueda ${ }^{3}$ \\ ${ }^{1}$ Department of Physics \& Astronomy, University of Leicester, Leicester, LE1 7RH, UK \\ e-mail: mgw@star.le.ac.uk \\ 2 Subaru Telescope, National Astronomical Observatory of Japan, 650 North A'ohoku Place, Hilo, HI 96720, USA \\ 3 Institute of Space and Astronautical Science, Sagamihara, Kanagawa 229-8510, Japan
}

Received 21 December 2004 / Accepted 6 April 2005

\begin{abstract}
We report the serendipitous discovery of several unresolved X-ray sources lying in the prominent spiral arms of the galaxy KUG 0214-057 in XMM-Newton observations. The location of these X-ray sources strongly suggests that at least three, and possibly four, of these may be physically related to the galaxy. The luminosity of each of these sources at the distance of KUG 0214-057 is $>5 \times 10^{39} \mathrm{erg} \mathrm{s}^{-1}(0.3-10 \mathrm{keV})$, making each a strong candidate ultraluminous X-ray source (ULX). Using the ULXs objects as a metric implies that this relatively low-mass galaxy may be experiencing rather intense starburst activity. The serendipitous discovery of these ULXs objects suggests that such objects are not a negligible component of the overall extragalactic X-ray source population.
\end{abstract}

Key words. X-rays: galaxies - galaxies: starburst

\section{Introduction}

Ultraluminous X-ray sources (ULXs) are the second most luminous class of discrete $\mathrm{X}$-ray sources associated with external galaxies, only surpassed in luminosity terms by active galactic nuclei (AGN). However, unlike AGN, they can be located anywhere within the confines of a galaxy. ULXs are observationally defined (e.g. Makishima et al. 2000) as discrete X-ray sources displaying a luminosity in excess of $10^{39} \mathrm{erg} \mathrm{s}^{-1}$, equal to the Eddington luminosity for accretion onto a $\sim 7 M_{\odot}$ black hole. Hence the high apparent luminosities of many ULXs, particularly those exceeding $10^{40} \mathrm{erg} \mathrm{s}^{-1}$, have led to a debate on their underlying nature (see Miller \& Colbert 2004 for a recent review). In particular, this debate focuses on whether any ULXs are powered by accretion onto "intermediate-mass" black holes (IMBH, with $M_{\mathrm{BH}} \sim 10^{2}-10^{5} M_{\odot}$; e.g. Colbert \& Mushotzky 1999; Miller et al. 2003), or whether instead accretion onto a stellar-mass black hole is responsible $\left(M_{\mathrm{BH}}<20 M_{\odot}\right.$, as seen for Galactic black holes), where the high luminosities originate either in truly super-Eddington radiation from inhomogeneous accretion discs (Begelman 2002), or through anisotropic radiation patterns and/or relativistic beaming (e.g. King et al. 2001; Reynolds et al. 1997).

Perhaps the most interesting result to emerge from Chandra observations of starburst galaxies has been the detection of

\footnotetext{
* Based on observations obtained with XMM-Newton, an ESA science mission with instruments and contributions directly funded by ESA Member States and NASA.
}

significant populations of ULXs, many of which have extreme luminosities i.e. close to or in excess of $10^{40} \mathrm{erg} \mathrm{s}^{-1}$, in the most active star forming regions of these galaxies (e.g. NGC 3256, Lira et al. 2002; the Antennae, Fabbiano et al. 2001; the Cartwheel, Gao et al. 2003). Whilst it is possible that some of these ULXs may be powered by IMBHs formed in young, dense star clusters (as now seems likely for the extreme ULX in M 82; Portegies Zwart et al. 2004), this formation route is unlikely for the majority of ULXs in these systems, which are instead likely to be "ordinary" high-mass X-ray binaries powered by one or more of the mechanisms outlined above (King 2004). Regardless of their origin, it is now becoming apparent that the presence (and integrated luminosity) of multiple ULXs may be a new gauge of high rates of star formation activity (Grimm et al. 2003; Persic et al. 2004), and indeed the numbers of galaxies containing ULXs has been demonstrated to rise proportionally with the star formation rate out to $z \approx 0.1$ (Hornschmeier et al. 2004).

Most of the ULXs known have been discovered in pointed observations of specific galaxies, i.e. essentially in surveys of external galaxy populations, although their discovery has often been fortuitous. In this paper we report the serendipitous discovery of several unresolved X-ray sources lying in the prominent spiral arms of the galaxy KUG 0214-057 in XMM-Newton observations of this region of sky, made as part of the Subaru XMM-Newton Deep Survey (SXDS). The location of these $\mathrm{X}$-ray sources strongly suggests that at least three, and possibly four, of these may be physically related to the galaxy as 

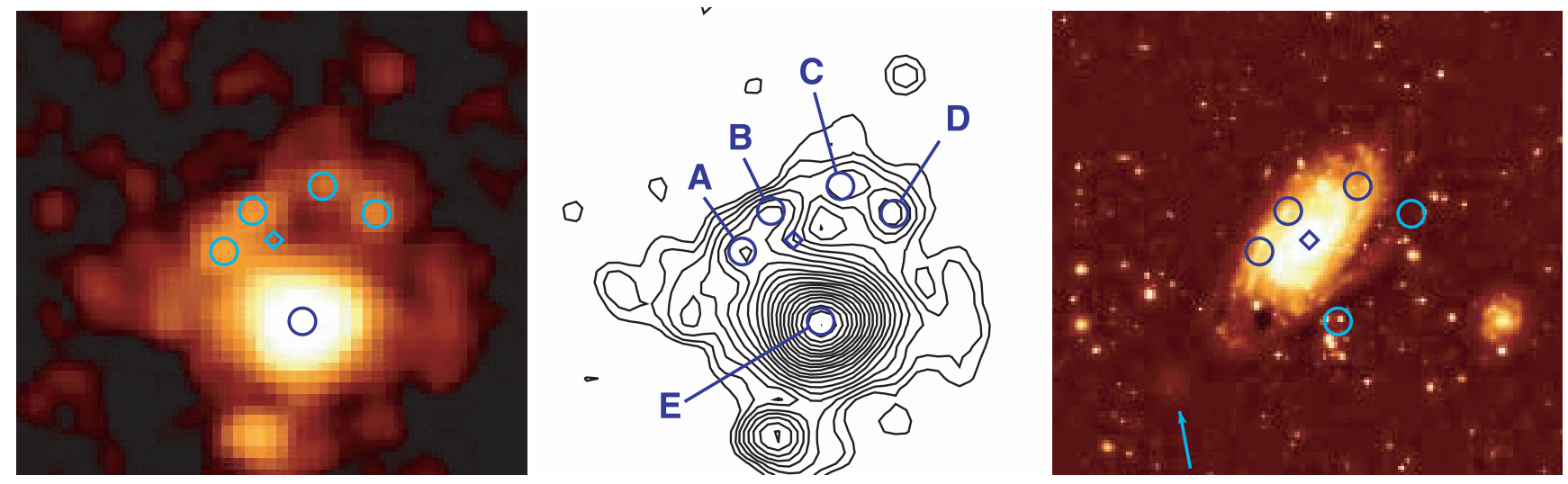

Fig. 1. a) and b) (left and centre panels): the soft band (0.5-2 keV) XMM-Newton X-ray image centred on KUG 0214-057. Data shown is for the 3 EPIC cameras combined in both colour and contour representations. The circles, plotted with nominal 5 arcsec radius, mark the 5 sources discussed in the text, labelled with source identifiers as in Table 1. Note that source E is strongly saturated in the image shown in a). The location of the nucleus of KUG 0214-057 is marked with a diamond in each panel. The X-ray image has been smoothed with a Gaussian with $\sigma=5$ arcsec. c) (right-hand panel): optical $R$-band image of region containing KUG 0214-057 obtained with Subaru SuprimeCam. The smaller galaxy to the E is APMUKS(BJ) B021449.37-054240.9; the nebulosity discussed in the text is the faint feature to the SE marked with an arrow. Each panel has dimensions $\sim 3 \times 3$ arcmin.

opposed to foreground or background objects. The luminosity of each of these sources, if at the distance of KUG 0214-057, is $>5 \times 10^{39} \mathrm{erg} \mathrm{s}^{-1}$, placing all three towards the high luminosity end of the ULX regime. These results thus correspond to the discovery of ULXs as part of an unbiased extragalactic survey.

\section{Observations and data analysis}

The X-ray observations constituting the SXDS comprise a mosaic of 7 partially overlapping pointings with XMM-Newton covering $\approx 1.2 \mathrm{sq} \mathrm{deg}$ with exposure times of $100 \mathrm{ks}$ (central field) and $50 \mathrm{ks}$ (flanking fields). The results presented here are drawn from the complete X-ray source catalogue for the SXDS region which contains $>1000$ objects (Ueda et al. 2005 , in preparation). The SXDS X-ray catalogue was produced standard XMM-Newton SAS tools and utilises an approach very similar to the standard pipeline processing (Watson et al. $2001^{1}$ ). Small improvements were employed, e.g. to optimise the background estimation and to ensure closely-spaced sources are correctly resolved.

Here we concentrate on the "SDS-6" field, which was observed for a total exposure time $\approx 50 \mathrm{ks}$. The SDS-6 field (OBSID: 0112370701, observations made on 2002-08-08/ 2002-08-09) contains a small region notable in having a relatively high local source density. Our attention was first drawn to this source complex because of the rather striking pattern: a single bright source surrounded by a partial ring of fainter objects (Fig. 1a,b). As is discussed later, there is no reason to suppose that the bright X-ray source and fainter objects are physically related, but it is very likely that several of the faint X-ray sources are physically related to a galaxy they are spatially coincident with.

Table 1 presents the basic parameters of the five X-ray sources detected by XMM-Newton in this region of sky taken

\footnotetext{
${ }^{1}$ See also the documentation for the $1 \mathrm{XMM}$ catalogue which uses a very similar procedure: http://xmmssc-www.star.le.ac.uk/
}

from the complete X-ray source catalogue for the SXDS (Ueda et al. 2005, in preparation). The catalogue is based on an analysis of the summed EPIC images, i.e. the EPIC MOS1, MOS2 and pn data combined; all the results discussed in this paper relate to the combined EPIC data. Source parameters are those derived from the SAS task emldetect which determines the source parameters by fitting the instrumental point spread function (PSF) at candidate source positions as part of the standard source detection and parameterisation approach. All 5 sources reported in Table 1 are detected at relatively high significance (likelihoods $\geq 30$ in the combined EPIC data; sources C \& D have the lowest likelihoods) and are confirmed by visual examination of the EPIC pn images and in the combined EPIC MOS images (the lowest significance sources are only marginally visible in the individual EPIC MOS images). The count rates reported are background-subtracted values corrected for vignetting and correspond to the count rates integrated over the entire instrument PSF.

Figures 1a,b show the XMM-Newton X-ray image with the source locations overlaid. By cross-correlating the full set of X-ray sources detected in the SXDS region with the Subaru optical object catalogue, the relative astrometry between the Subaru and XMM-Newton astrometric frames has been established to $<1$ arcsec. The positions quoted in Table 1 have been corrected for small systematic shifts in the XMM-Newton astrometric frame, i.e. essentially to the reference frame defined for the Subaru imaging which has an absolute astrometric accuracy better than 0.5 arcsec. The statistical error $(1 \sigma)$ on the X-ray source positions is $<0.5$ arcsec for the brightest source (E) and ranges from 1.2 to 1.8 arcsec for the four fainter sources listed. We adopt a 5 arcsec radius for the X-ray source positional uncertainty.

The location of the X-ray sources on the optical sky is shown in Fig. 1c. The optical image shown is the $R$-band image obtained as part of the Subaru SuprimeCam observations of the SXDS. The full SXDS optical imaging consists 
Table 1. Summary properties of the X-ray sources in the region of KUG 0214-057.

\begin{tabular}{cccccccccc}
\hline \hline $\begin{array}{c}\text { Source }^{a} \\
\text { name }\end{array}$ & RA & Dec & $\begin{array}{c}\text { Count rate }^{b} \\
\text { [count s }\end{array}$ ] & HR1 & HR2 & HR3 & $\log _{10} f_{\mathrm{X}}{ }^{d}$ & $z^{e}$ & $\log _{10} L_{X}{ }^{f}$ \\
\hline A & 021720.60 & -052855.1 & $0.00499 \pm 0.00043$ & $0.83 \pm 0.07$ & $-0.59 \pm 0.07$ & $-0.59 \pm 0.29$ & -13.87 & 0.018 & 39.98 \\
B & 021719.88 & -052839.8 & $0.00377 \pm 0.00039$ & $0.81 \pm 0.08$ & $-0.59 \pm 0.07$ & $-0.41 \pm 0.34$ & -13.99 & 0.018 & 39.86 \\
C & 021718.13 & -052830.5 & $0.00370 \pm 0.00044$ & $0.63 \pm 0.09$ & $-0.68 \pm 0.08$ & $-0.25 \pm 0.38$ & -14.00 & 0.018 & 39.85 \\
D & 021716.77 & -052840.8 & $0.00245 \pm 0.00036$ & $1.00 \pm 0.04$ & $-0.50 \pm 0.12$ & $-0.44 \pm 0.37$ & -14.17 & 0.018 & 39.67 \\
E & 021718.62 & -052921.3 & $0.12428 \pm 0.00131$ & $0.57 \pm 0.01$ & $-0.73 \pm 0.01$ & $-0.42 \pm 0.02$ & -12.47 & 0.634 & 44.76 \\
\hline
\end{tabular}

${ }^{a}$ IAU designations of sources are as follows: A XMMU J021729.6-052855; B XMMU J021719.9-052840; C XMMU J021718.1-052831; D XMMU J021716.8-052841; E XMMU J021718.6-052921.

${ }^{b} \mathrm{X}$-ray count rate in $0.3-10 \mathrm{keV}$ band expressed in EPIC pn count $\mathrm{s}^{-1}$.

${ }^{c}$ Hardness ratios HR1, HR2 and HR3 are defined in the caption to Fig. 3.

${ }^{d} \mathrm{X}$-ray flux in $0.3-10 \mathrm{keV}$ band [erg $\mathrm{cm}^{-2} \mathrm{~s}^{-1}$ ] assuming a nominal power-law spectrum with $\Gamma=1.7$ and absorption column density $N_{\mathrm{H}}=2.5 \times 10^{20} \mathrm{~cm}^{-2}$.

${ }^{e}$ Assumed redshift (see text).

${ }^{f} \mathrm{X}$-ray luminosity in $0.3-10 \mathrm{keV}$ band $\left[\mathrm{erg} \mathrm{s}^{-1}\right]$.
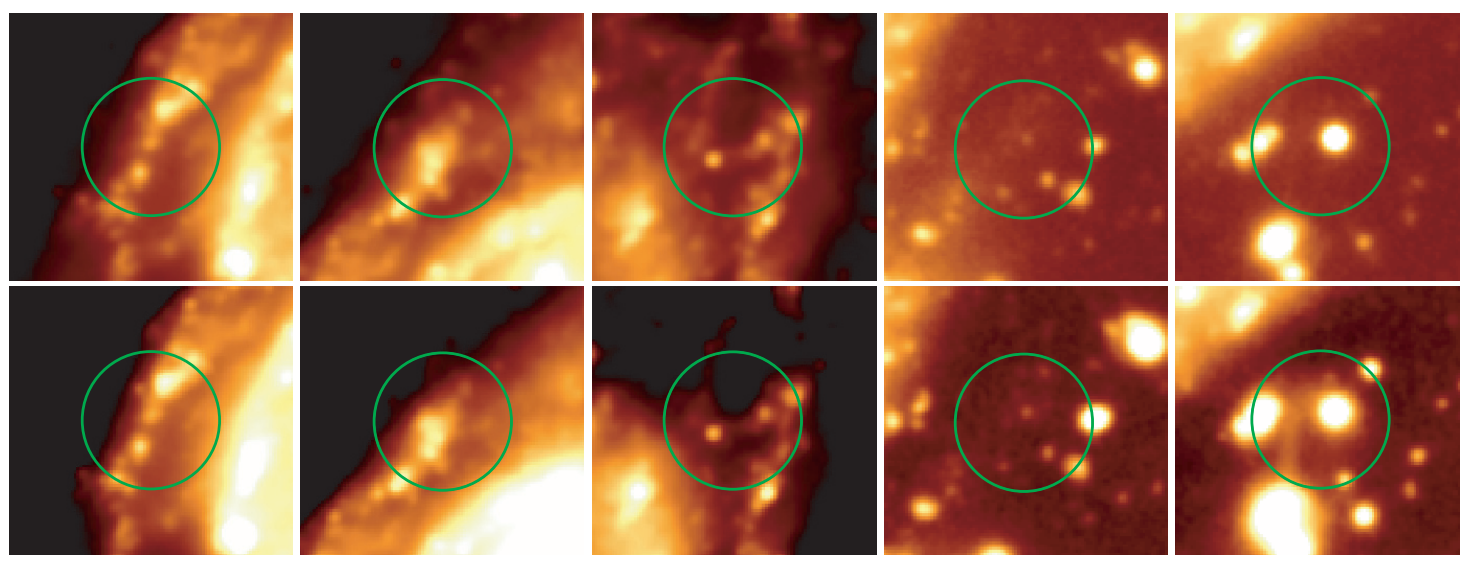

Fig. 2. Optical $B$-band (top row) and $R$-band (bottom row) finding charts for the four ULX candidate X-ray sources (A, B, C, D from left to right) and the background AGN (E). The finding charts are based on the Subaru SuprimeCam SXDS imaging. X-ray error-circles, plotted with nominal 5 arcsec radius, are shown in green. Note that the two right hand images are displayed with different colour table to enhance the visibility of faint objects.

of five colours $\left(B V R i^{\prime} z^{\prime}\right)$ and extends to very faint limits (e.g. $B \approx 27.5 \mathrm{mag} ; \mathrm{AB} ; 5 \sigma)$ with excellent seeing $(\approx 0.8 \operatorname{arcsec})$ (Furusawa et al. 2005, in preparation).

\section{Results and discussion}

\subsection{Location and nature of the $X$-ray sources}

As can be seen in Fig. 1c, all five ${ }^{2} \mathrm{X}$-ray sources lie in the outer regions of the 15 mag galaxy KUG 0214-057 (Miyauchi-Isobe \& Mahehara 1998; KUG 0214-057 = MCG -01-06-080/ PGC 008726). KUG 0214-057 is a barred spiral galaxy at $z=$ 0.018 (corresponding to a distance $D \approx 75 \mathrm{Mpc}$ ), lying in the galaxy group NOG 151 with NGC 881 and NGC 883 (Giuricin et al. 2000). Three of these sources (A, B and C) are located in

\footnotetext{
${ }^{2}$ Note: Fig. 1a,b shows a sixth bright X-ray source at the lower edge of the image. This is identified with an AGN at $z=0.38$ and is hence not connected with the galaxy.
}

the outer spiral arms of the galaxy, whilst the other two (E, D) are outside the visible optical envelope.

The brightest source, E, has been identified with an AGN at $z=0.634$ as part of the ongoing optical follow-up of the SXDS X-ray sources (Akiyama et al. 2005, in preparation); the optical counterpart is the brightest object within the X-ray error-circle shown in Fig. 2. As KUG 0214-057 is at $z=0.018$, the X-ray source E is clearly an unrelated background object ${ }^{3}$.

The location of the remaining four objects is most plausibly explained by them being located within the galaxy itself. The evidence is arguably strongest for sources A, B and C which are embedded in regions of the galaxy rich in knots of nebulosity which, on the basis of their broad-band optical colours, are very likely to be star-formation regions (see Fig. 2 and Sect. 3.3). The evidence is weaker for $\mathrm{D}$ which lies somewhat

\footnotetext{
3 We note that the $R$-band finding chart for source E (Fig. 2) shows evidence for diffuse emission, including an unusual "bridge" feature, which may indicate the AGN galaxy is part of an interacting group.
} 

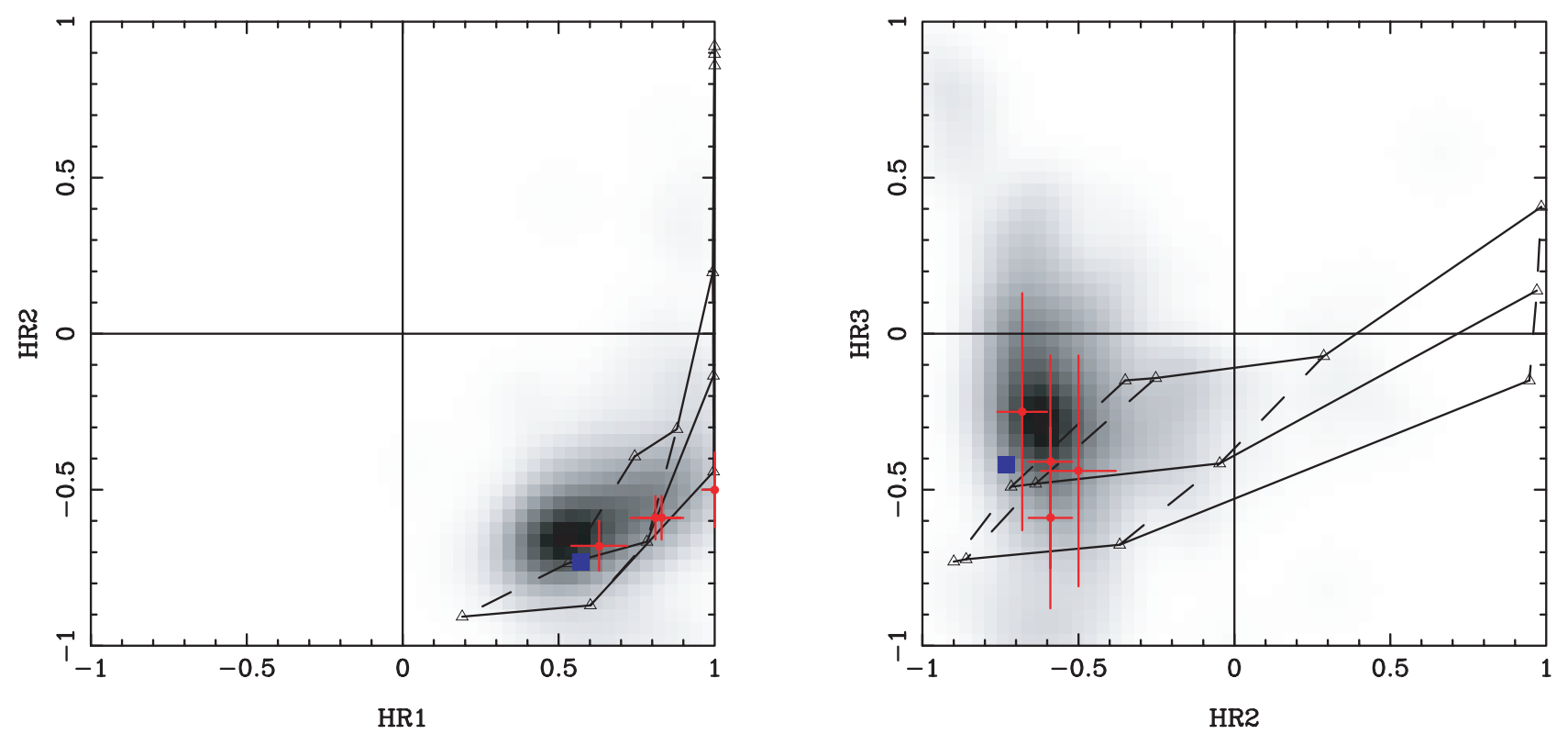

Fig. 3. X-ray colour-colour plots for the five X-ray sources: the four ULX candidates (red points and error bars) and the source identified with an AGN (E; blue square, error bars are $\sim 2$ times smaller than the symbol plotted). The background grey-scale shows the density of colour values for $\sim 500$ SXDS X-ray sources with relatively low errors in the X-ray colour. Left: HR2 vs. HR1; right: HR3 vs. HR2. The HR values are defined as follows: $\mathrm{HR} 1=(\mathrm{B}-\mathrm{A}) /(\mathrm{A}+\mathrm{B}) ; \mathrm{HR} 2=(\mathrm{C}-\mathrm{B}) /(\mathrm{B}+\mathrm{C}) ; \mathrm{HR} 3=(\mathrm{D}-\mathrm{C}) /(\mathrm{C}+\mathrm{D})$ where $\mathrm{A}=C_{\mathrm{X}}(0.3-0.5 \mathrm{keV}) ; \mathrm{B}=C_{\mathrm{X}}(0.5-2 \mathrm{keV})$; $\mathrm{C}=C_{\mathrm{X}}(2-4.5 \mathrm{keV})$ and $\mathrm{D}=C_{\mathrm{X}}(4.5-10 \mathrm{keV})$ and the $C_{\mathrm{X}}$ values are the vignetting-corrected EPIC count rates. The grid overlaid on each panel corresponds to the hardness ratios for power law spectra with indexes $\Gamma=[1,2,3]$ (top to bottom) and $N_{\mathrm{H}}=\left[10^{20}, 10^{21}, 10^{22}, 10^{23}\right] \mathrm{cm}^{-2}($ left to right).

outside the visible optical envelope of the galaxy, although not far from the end of the apparently disrupted spiral arm to the $\mathrm{W}$. If this is correct, the broad band X-ray luminosities of these sources range from $\sim 5 \times 10^{39} \mathrm{erg} \mathrm{s}^{-1}$ to $\sim 10^{40} \mathrm{erg} \mathrm{s}^{-1}$, making them ULXs with luminosities towards the high end of those observed in external galaxies.

These X-ray sources might alternatively be foreground or background objects unrelated to the galaxy. Based on the wellestablished high Galactic latitude X-ray source $\log N-\log S$ relationship, the a priori chance probability of finding three or four X-ray sources at these fluxes within 1 arcmin radius of the galaxy is $<1 \%$ and the probability of finding them in the smaller region defined by the spiral arms even lower. More than $80 \%$ of faint high latitude X-ray sources are AGN, making them the most likely background sources. At the flux levels of the four sources under discussion, the AGN counterparts have mean optical magnitude $R \sim 23-24$ mag. Although counterparts at these magnitudes are normally trivially detected in the Subaru imaging observations, we estimate that the surface brightness inside the envelope of KUG 0214-057 is high enough to preclude detection of counterparts fainter than $\sim 22$ mag. Thus only a small fraction of potential background AGN counterparts would actually be detectable.

\section{2. $X$-ray properties of the ULX candidates}

The four ULX candidates discussed above are too faint for detailed X-ray spectroscopy, but their X-ray colours can nevertheless provide some constraints on the continuum shape and low energy absorption. Figure 3 shows the X-ray colours for the ULX candidates and, for comparison, the distribution of hardness values for other X-ray sources in the SXDS which is of course dominated by AGN in the field. Also shown is the position of E, the source identified with a luminous AGN. Overall the four ULX candidates have slightly higher values of HR1 than the average of all sources in the SXDS field and somewhat lower values of HR3 than the average ${ }^{4}$. However the suggested power-law indices of $\Gamma \sim 2$ and low-to-moderate columns $\left(\sim 10^{21} \mathrm{~cm}^{-2}\right)$ of sources $\mathrm{C}, \mathrm{A}$ and B are quite typical for accretion-powered ULXs (e.g. Roberts et al. 2004; Colbert et al. 2004; Swartz et al. 2004). Only source D appears unusual, with a suggestion of a higher column and softer power-law index (Fig. 3 left panel). Since this source lies away from the main galaxy disc, and has a potential red counterpart, this may indicate that it is a background source, though an intriguing possibility is that its X-ray colours could be explained by a "buried" supernova remnant (cf. source CXOU J123029.5+413927 in NGC 4490, Roberts et al. 2002).

A second X-ray diagnostic of the nature of these sources is temporal variability. This is a particularly important test for relatively distant sources such as these ULX candidates, where

\footnotetext{
${ }^{4}$ The location of these sources in the X-ray hardness ratio plot (Fig. 3) demonstrates that the nominal spectrum used to compute the fluxes and luminosities (Table 1) is a reasonable assumption given the quality of the data. The derived fluxes and luminosities are of course sensitive to the spectral assumptions: increasing the assumed column density to $N_{\mathrm{H}} \sim 10^{21} \mathrm{~cm}^{-2}$ would increase them by a factor of $\sim 1.5$, whilst the possible range of spectral indices also introduces similar changes in the derived values, with steeper (flatter) spectra reducing (increasing) the flux and luminosity estimates by factors $\sim 1.5-2$.
} 
the spatial resolution of XMM-Newton corresponds to physical scales of $\sim 2 \mathrm{kpc}$, hence a detection of variability (particularly on short timescales) strongly favours an origin for the $\mathrm{X}$-ray emission in a single accreting source as opposed to an unresolved complex of many sources. However, the limited nature of the raw data (100-200 counts per ULX candidate from all three EPIC detectors combined) precludes all but the simplest of variability tests. Comparative photometry of the four ULX candidates, after splitting the observation into three equal-length intervals ( $\sim 15.9 \mathrm{ks}$ apiece), revealed no significant variability. A Kolmogorov-Smirnov (K-S) test against the hypothesis of a constant source flux for the time series data divided into $5 \mathrm{ks}$ intervals indicated no significant variability in three of the sources, but source B was found to have a $~ 98 \%$ probability of being variable $(\sim 2.4 \sigma$ significance). Hence at least one of the ULX candidates shows some temporal evidence of being a single, accretion-powered source.

The X-ray properties of sources A \& $\mathrm{C}$ and in particular B appear consistent with known ULXs. Source D has slightly unusual X-ray colours, perhaps consistent with a buried young supernova remnant, which would make it a very rare source - only $\sim 10$ such sources with peak X-ray luminosities in the ULX regime have ever been observed (Immler \& Lewin 2003; this rarity also argues that the chances of the other ULX candidates also being such sources is very low indeed). There is of course still a possibility that some of the candidate ULXs are no more than a superposition of less-luminous sources clustered within the $\sim 5$ arcsec diameter XMM-Newton beam ( $\equiv 1.8 \mathrm{kpc}$ at the distance of KUG 0214-057). However, this would require a remarkable density of X-ray sources - for illustration, the surface luminosity density of the 43 detected $\mathrm{X}$-ray sources in the central 1 -arcmin ( $\equiv 5.5 \mathrm{kpc}$ diameter) region of the Antennae, calculated from the catalogue of Zezas et al. 2002 expressed as $\Sigma L_{\mathrm{X}} /$ area, is $\sim 9.5 \times 10^{38} \mathrm{erg} \mathrm{s}^{-1} \mathrm{kpc}^{-2}$. In KUG 0214-057 one would require a higher density of $1.6-3 \times 10^{39} \mathrm{erg} \mathrm{s}^{-1} \mathrm{kpc}^{-2}$ for any of our candidate ULXs to be a superposition of many fainter sources. Hence the most likely interpretation must be that we are viewing individual, accretion-powered ULXs.

\subsection{Optical spectroscopy}

An optical spectrum of the brightest optical feature lying within the X-ray error-circle for source B (second top and bottom panels in Fig. 2) was obtained with the Subaru FOCAS instrument in clear conditions with 1 arcsec seeing on 25/12/2003 in an observation lasting a total of $1 \mathrm{~h}$ (Akiyama et al. 2005, in preparation). The observation employed the $150 \mathrm{~mm}^{-1}$ low-resolution grating and the SY47 filter to minimise order overlap, providing wavelength coverage from 4700 to $9400 \AA$ with resolving power $R=250$. The resultant reduced spectrum is shown in Fig. 4. The line ratios for $[\mathrm{SII}] / \mathrm{H} \alpha$ and $[\mathrm{OIII}] / \mathrm{H} \beta$ demonstrate that this is the spectrum of an HII region rather than an AGN, albeit one with relatively high excitation when compared with other Galactic and extragalactic examples (e.g. Kennicutt et al. 2000). In fact the observed ratios are close to HII/AGN dividing line, indicating that a hard X-ray photoionizing source may

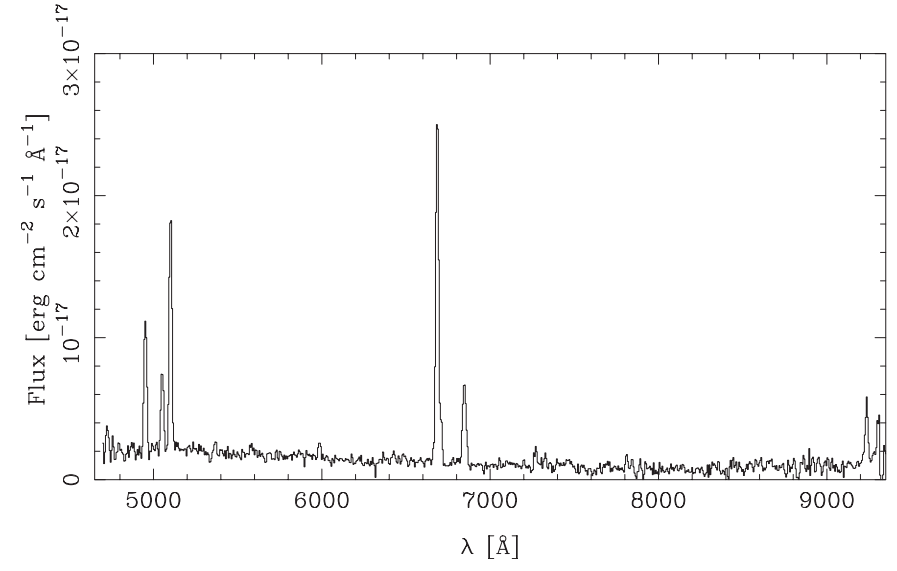

Fig. 4. FOCAS optical spectrum of brightest knot in nebulosity within error-circle of source B (see Fig. 2). The bright emission lines are identified as $\mathrm{H} \alpha(+[\mathrm{NII}]),[\mathrm{OIII}] \lambda 4959,5007 \AA, \mathrm{H} \beta,[\mathrm{SII}] \lambda 6716,6731 \AA$ and [SIII] $\lambda 9069 \AA$ at $z=0.018$.

be located in the nebula as expected for a ULX (e.g. Pakull \& Mirioni 2003; Roberts et al. 2003) although it is doubtful that source B could provide all the ionising flux.

\subsection{Nature and properties of the galaxy KUG 0214-057}

From its optical appearance, KUG 0214-057 is clearly a late-type galaxy with very significant recent star formation. Its inclusion in the KUG catalogue of UV-excess galaxies reinforces this view as UV-excess is most commonly an indicator of relatively strong star formation. There is some indication of disruption to the outer spiral arms. It is also interesting to note the small companion galaxy (APMUKS(BJ) B021449.37-054240.9) 1.5 arcmin. to the SW which may be interacting with KUG 0214-057, and the very faint nebulosity lying a similar distance to the SE (see Fig. 1c). This nebulosity might actually be a fragment left over from an earlier interaction.

KUG 0214-057 was not detected by IRAS and hence there is no mid-IR data to constrain the star formation rate. Similarly, there are no corresponding measurements of the flux in $\mathrm{H} \alpha$, UV or radio continuum, hence these classic methods for deriving a star forming rate are also not available. However, recent work has shown that the integrated X-ray luminosity of luminous high mass $\mathrm{X}$-ray binaries in star forming galaxies can also be a measure of star formation rate (see e.g. Grimm et al. 2003). We therefore estimate an X-ray star forming rate in the following manner. We assume a starburst-like X-ray luminosity function slope of $\gamma=0.5$ (Kilgard et al. 2002) for sources in KUG 0214-057, and normalise this to the integrated flux of the four detected sources $\left(\sim 2.8 \times 10^{40} \mathrm{erg} \mathrm{s}^{-1}\right)$ over the $4-20 \times 10^{39} \mathrm{erg} \mathrm{s}^{-1}$ range (i.e. between the minimum detected luminosity of the ULXs, and the apparent break luminosity for galaxian luminosity functions noted by Grimm et al. 2003). We then integrate below this range (i.e. between $0-4 \times 10^{39} \mathrm{erg} \mathrm{s}^{-1}$ ) to estimate the "missing" flux from discrete sources below our 
detection limit ${ }^{5}$, which totals $2.3 \times 10^{40} \mathrm{erg} \mathrm{s}^{-1}$. Hence we estimate a total X-ray flux from luminous point-like $\mathrm{X}$-ray sources of $\sim 5 \times 10^{40} \mathrm{erg} \mathrm{s}^{-1}$. From Fig. 7 of Grimm et al. (2003) this converts to a reasonably high star formation rate in the range of $\sim 3.5-6 M_{\odot} \mathrm{yr}^{-1}$.

To put this star formation rate in perspective, we estimate the mass of KUG 0214-057 using the stellar $M / L$ correlation coefficients of Bell \& De Jong (2001). In particular, we follow Colbert et al. (2004) and use coefficients from the formation with bursts model, and we use optical/IR magnitudes from NED of $B=15.26$ (Maddox et al. 1990) and $K=13.16$ (2MASS measurement). These convert to give $\log _{10} L_{K} \approx 42.3 \mathrm{erg} \mathrm{s}^{-1}, \log _{10}\left(M / L_{K}\right) \approx 0.25$ and hence $M \approx 1.2 \times 10^{10} M_{\odot}$. Finally, by normalising the star formation rate to the galaxy mass we find that KUG 0214-057 has a rate of $\sim 4$ (in units of $10^{-10} \mathrm{yr}^{-1}$ ), which from Table 1 of Grimm et al. (2003) is very similar to the rate per unit mass in the archetypal local starburst M $82(\sim 3.6)$. However, in contrast to M 82, the locations of both the ULXs and optical knots demonstrate that the star formation activity appears to be prevalent throughout the spiral arms of KUG 0214-057, rather than dominant in the nucleus. This is another indicator that the high star formation rate is due to an interaction: similar star formation morphology and ULX locations are seen in more nearby interactions, e.g. in NGC 4485/90 (Roberts et al. 2002) and M51 (Terashima \& Wilson 2004). However, we note that both the star formation rates and the integrated and peak luminosities of the X-ray source populations are far higher in KUG 0214-057. Indeed, Ptak \& Colbert (2004) estimate that only $\sim 7 \%$ of galaxies possess one or more ULXs with $L_{\mathrm{X}}>2 \times 10^{39} \mathrm{erg} \mathrm{s}^{-1}$; KUG $0214-057$ potentially has four. This high incidence of ULXs is only bettered by the most intense local starbursts, usually in galaxy collisions e.g. NGC 3256 (Lira et al. 2002), the Cartwheel (Gao et al. 2003).

\section{Conclusions}

We have shown that the galaxy KUG $0214-057$ probably contains up to four ULXs with luminosities at the high end for such objects. Given the low mass of inferred for this galaxy, the high star formation implied by the presence of these ULXs indicates that this previously relatively obscure galaxy possesses remarkably intense starburst activity. These ULXs, discovered in the SXDS, are drawn from an unbiased extragalactic survey rather than pointed observations of specific galaxies, allowing an estimate to be made (albeit very crude) of the contribution ULXs make to the overall extragalactic source population. The three or four sources reported here comprise $\sim 0.5 \%$ of the

\footnotetext{
5 We caution that the actual detection limit could be lower, which would lower the estimated contribution from undetected sources, for example if the detection limit is $2 \times 10^{39} \mathrm{erg} \mathrm{s}^{-1}$ then the estimated missing flux reduces to $1.3 \times 10^{40} \mathrm{erg} \mathrm{s}^{-1}$ On the other hand, the PSF wings of the bright QSO obscure our view of the southern regions of the galaxy, hence we potentially miss detecting sources in this region which could lead to us underestimating the luminosity of the galaxy. Indeed there is clear evidence that the profile of source $\mathrm{E}$ is asymmetric (Fig. 1), implying the presence of one or more further faint objects in the vicinity of source E.
}

total source population detected in the SXDS. Several other ULX candidates exist in the SXDS (these have yet to be fully explored), demonstrating that this may be an underestimate of their total contribution. ULXs are thus a minority but not negligible population in deep surveys.

Acknowledgements. This work is based in part on data collected at Subaru Telescope, which is operated by the National Astronomical Observatory of Japan, financially supported in part by a Grant-in-Aid for Scientific Research (No. 15740126) by the Japanese Ministry of Education, Culture, Sports, Science and Technology.

\section{References}

Begelman, M. C. 2002, ApJ, 568, L97

Bell, E. F., \& de Jong, R. S. 2001, ApJ, 550, 212B

Colbert, E. J. M., \& Mushotzky, R. F. 1999, ApJ, 519, 89

Colbert, E. J. M., Heckman, T. M., Ptak, A. F., \& Strickland, D. K. 2004, ApJ, 602, 231

Fabbiano, G., Zezas, A., \& Murray, S. S. 2001, ApJ, 554, 1035

Gao, Y., Wang, Q. D., Appleton, P. N., \& Lucas, R. A. 2003, ApJ, 596, L171

Giuricin, G., Marinoni, C., Ceriani, L., \& Pisani, A. 2000, ApJ, 543, 178

Grimm, H.-J., Gilfanov, M., \& Sunyaev, R. 2003, MNRAS, 339, 793

Hornschemeier, A. E., Alexander, D. M., Bauer, F. E., et al. 2004, ApJ, 600, L147

Immler, S., \& Lewin, W. H. G. 2003, in Supernovae and Gamma-ray bursters, ed. K. Weiler, Lect. Notes Phys., 598, 91

Kennicutt, R. C., Bresolin, F., French, H., \& Martin, P. 2000, ApJ, 537, 589

Kilgard, R. E., Kaaret, P., Krauss, M. I., et al. 2002, ApJ, 573, 138

King, A. 2004, MNRAS, 347, L18

King, A., Davies, M. B., Ward, M. J., Fabbiano, G., \& Elvis, M. 2001, ApJ, 552, L109

Lira, P., Ward, M. J., Zezas, A., Alonso-Herrero, A., \& Ueno, S. 2002, MNRAS, 330, 259

Maddox, S. J., Sutherland, W. J., Efstathiou, G., \& Loveday, J. 1990, MNRAS, 243, 692

Makishima, T., Kubota, A., Mizuno, T., et al. 2000, ApJ, 535, 632

Miller, J. M., Fabbiano, G., Miller, M. C., \& Fabian, A. C. 2003, ApJ, $585, \mathrm{~L} 40$

Miller, M. C., \& Colbert, E. J. M. 2004, Int. J. Mod. Phys. D, 13, 1

Miyauchi-Isobe, N., \& Maehara, H. 1998, Publ. Nat. Astron. Obs. Japan, 5, 75

Pakull, M. W., \& Mirioni, L. 2003, Rev. Mex. Astron. Astrofis., 15, 197

Persic, M., Rephaeli, Y., Braito, V., et al. 2004, A\&A, 419, 849

Portegies Zwart, S. F., Baumgardt, H., Hut, P., Makino, J., \& McMillan, S. L. W. 2004, Nature, 428, 724

Ptak, A., \& Colbert, E. 2004, ApJ, 606, 291

Reynolds, C. S., Loan, A. J., Fabian, A. C., et al. 1997, MNRAS, 286, 349

Roberts, T. P., Warwick, R. S., Ward, M. J., \& Murray, S. S. 2002, MNRAS, 337, 677

Roberts, T. P., Goad, M. R., Ward, M. J., \& Warwick, R. S. 2003, MNRAS, 342, 709

Roberts, T. P., Warwick, R. S., Ward, M. J., \& Goad, M. R. 2004 MNRAS, 349, 1193

Swartz, D. A., Ghosh, K. K., Tennant, A. F., \& Wu, K. 2004, ApJS, 154,519

Terashima, Y., \& Wilson, A. S. 2004, ApJ, 601, 735

Watson, M. G., Auguères, J.-L., Ballet, J., et al. 2001, A\&A, 365, L51

Zezas, A., Fabbiano, G., Rots, A. H., \& Murray, S. S. 2002, ApJS, 142,239 